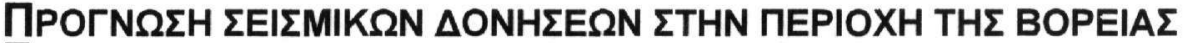

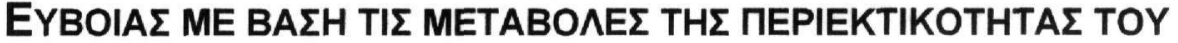 PADONIOY $\Sigma T A$ YMOTEIA NEPA.
}

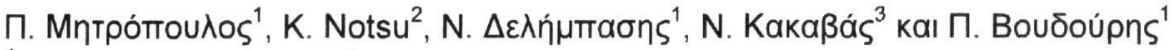

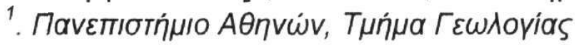 \\ 2. University of Tokyo, Department of Earthquake Chemistry

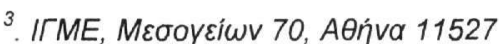

\section{ПЕРІ^НЧН}

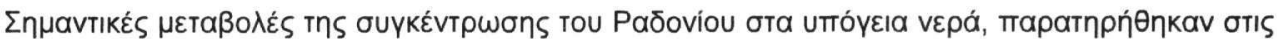

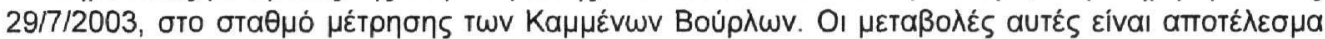

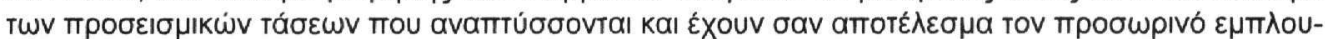

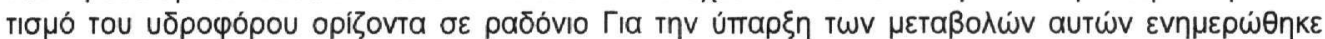

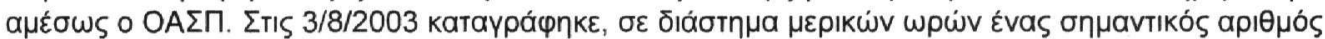

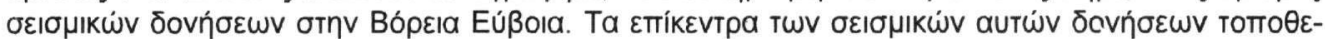

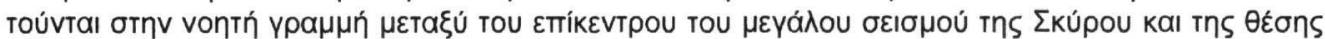

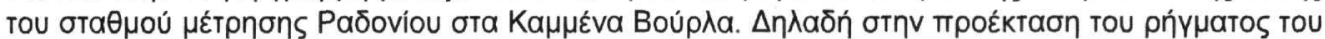
Bopzíou Aivaíou.

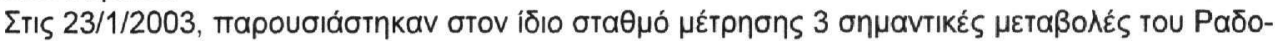

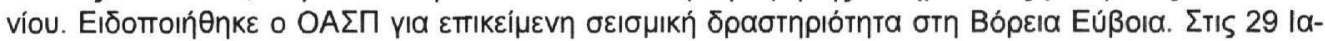

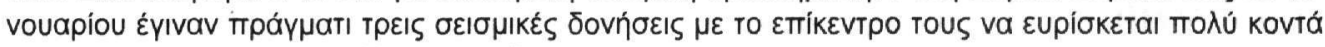

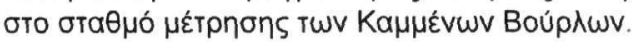

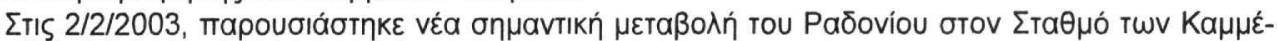

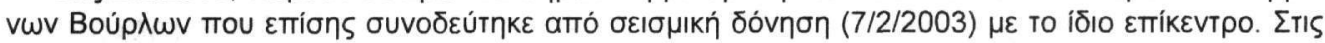

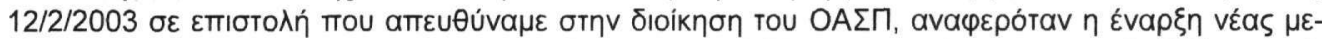

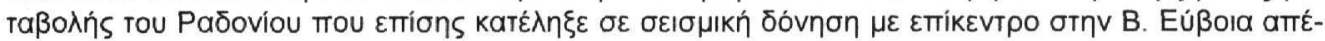

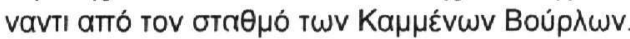

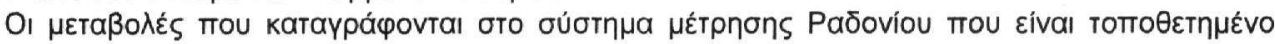

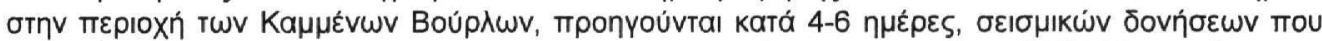

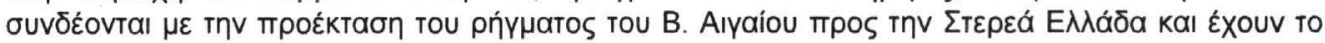

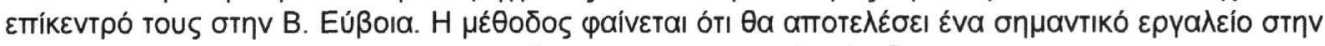

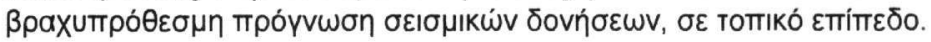

\section{$1 \operatorname{EI\Sigma AГ\Omega \Gamma H~}$}

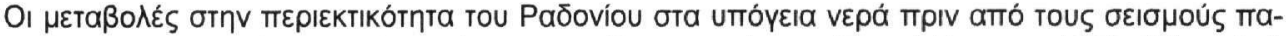

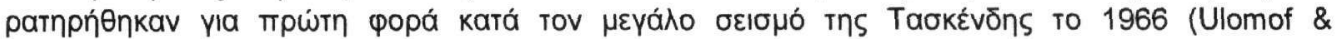

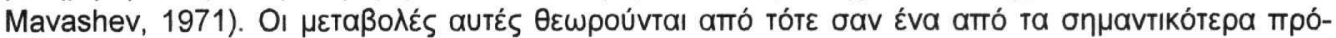

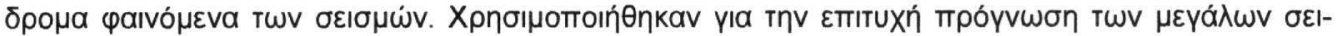

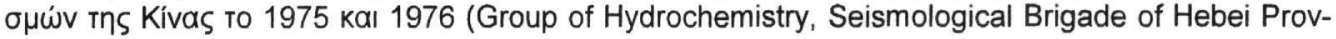
ince, 1976).

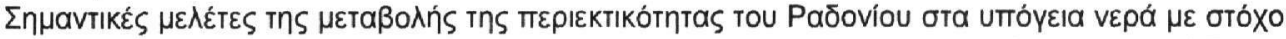

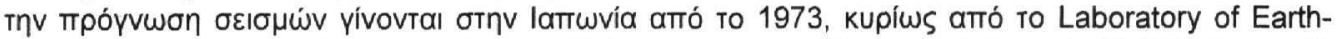

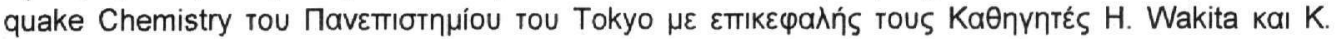

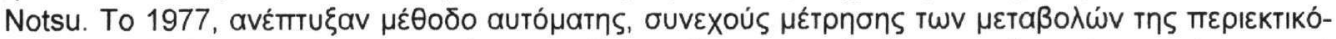

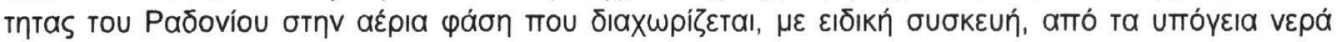




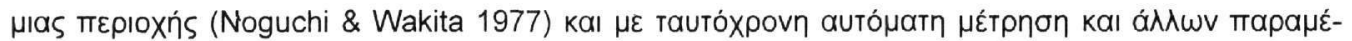

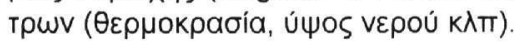

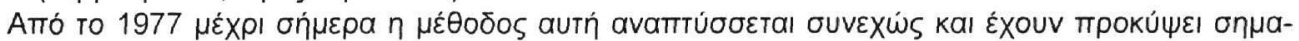

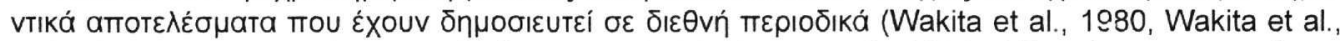

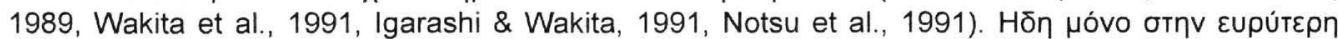

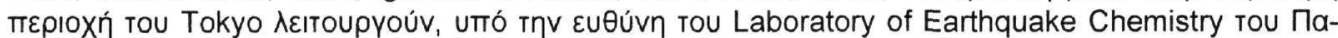

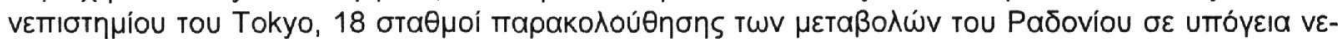

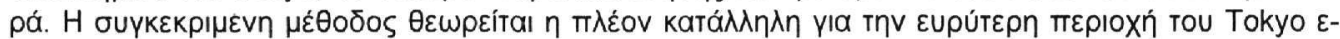

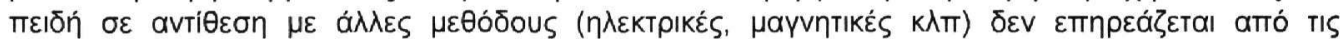

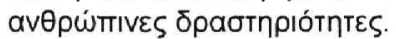

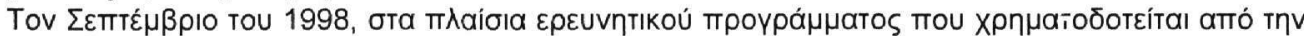

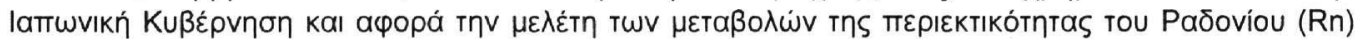

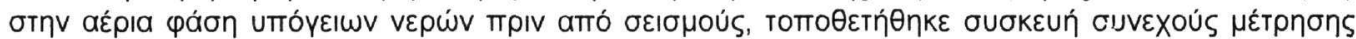

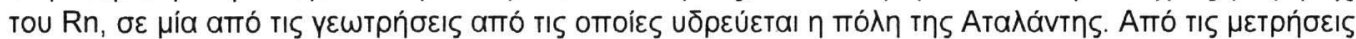

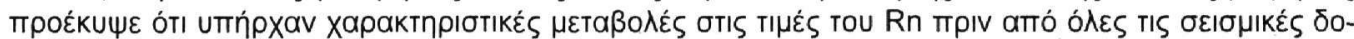

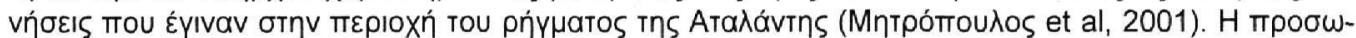

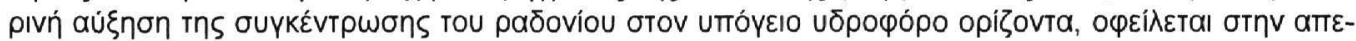

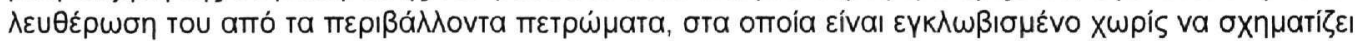

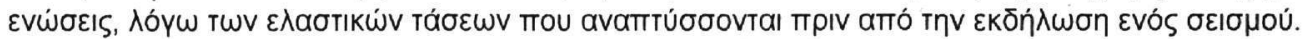

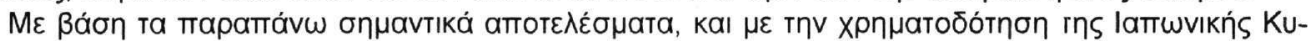

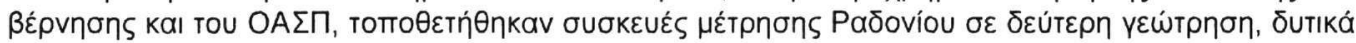

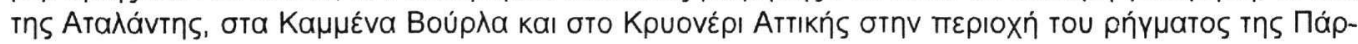
vnӨas.

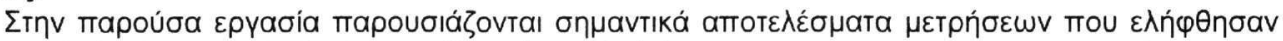

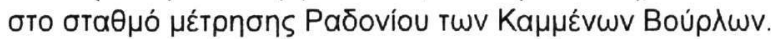

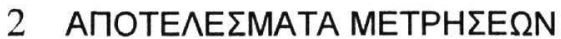

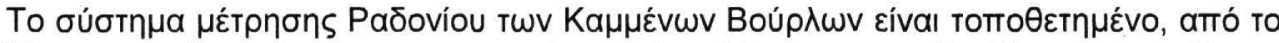

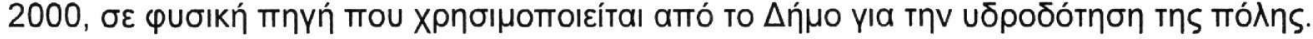

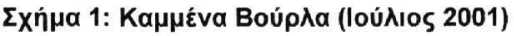

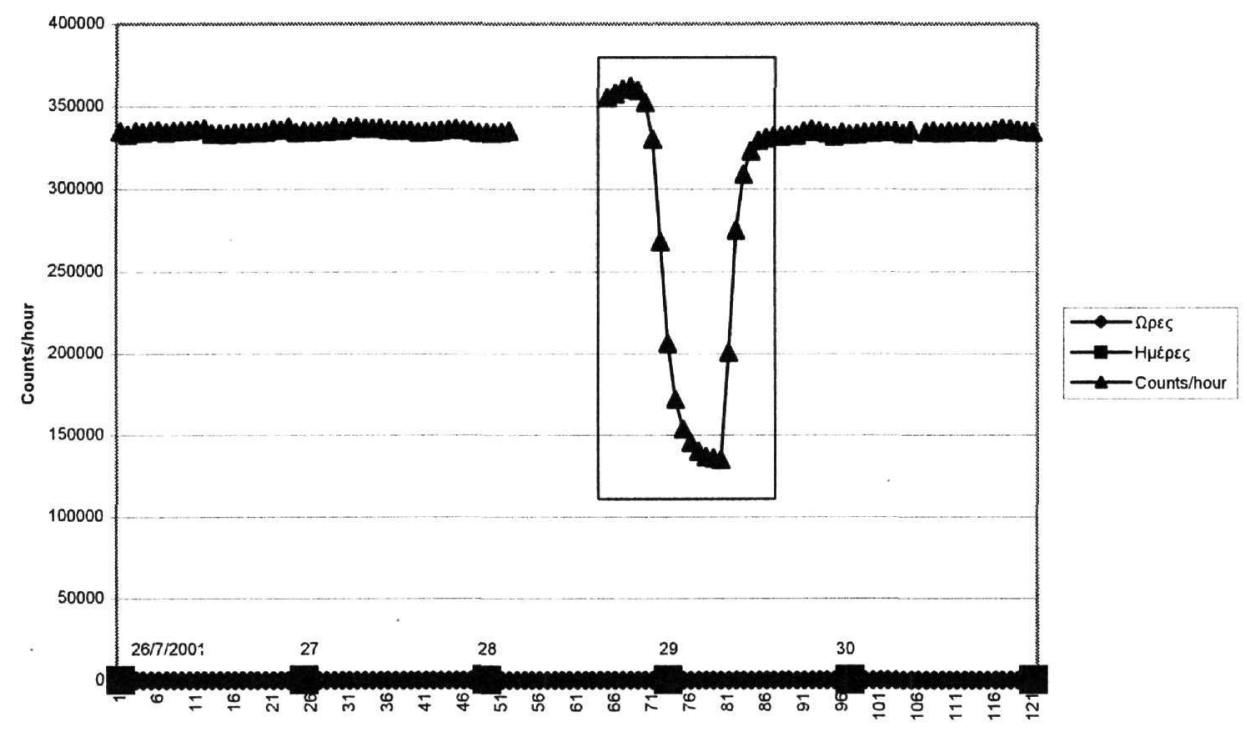




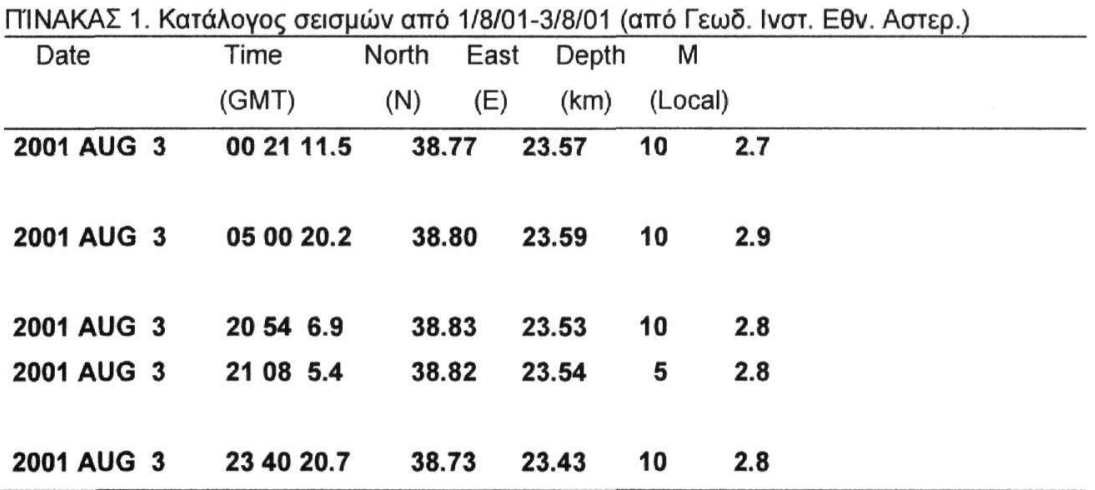

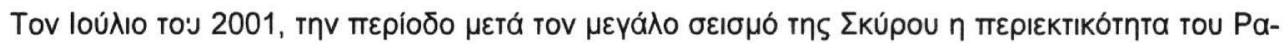

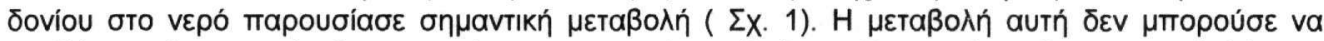

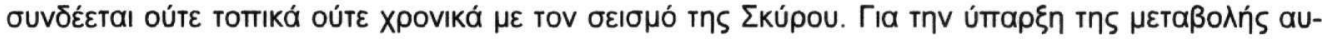

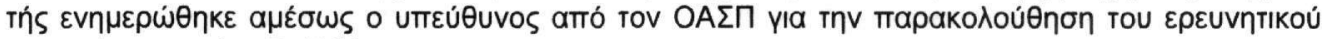

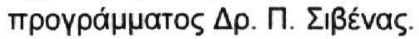

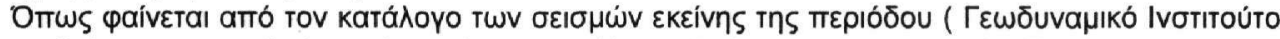

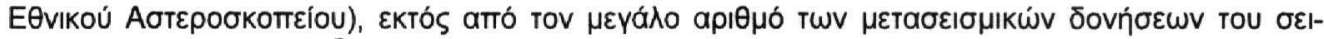

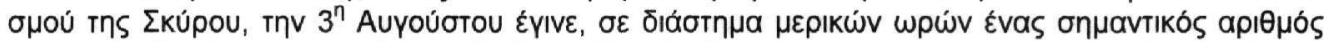

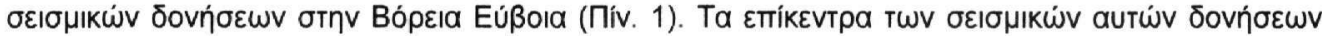

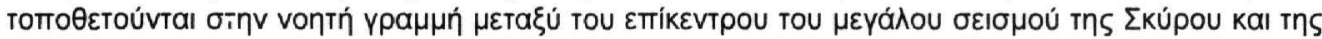

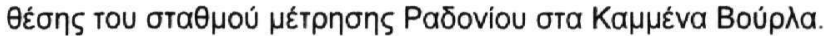

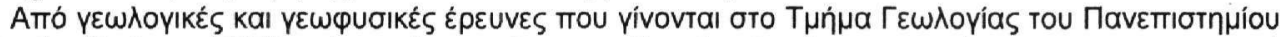

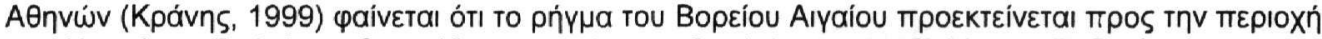

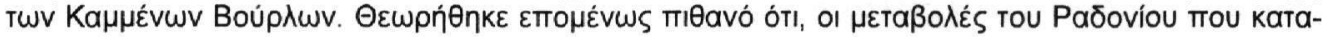

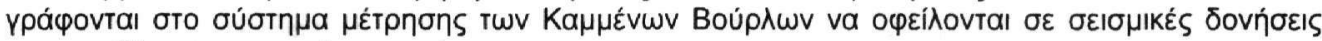

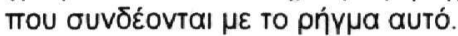

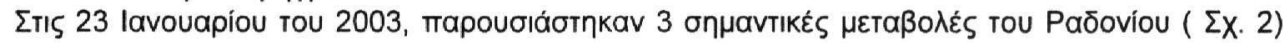

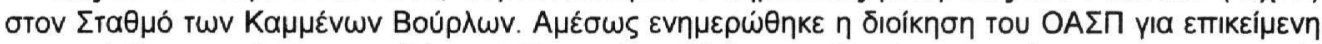

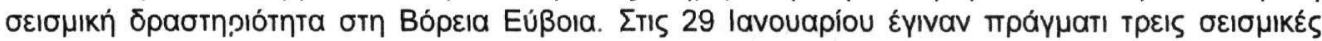

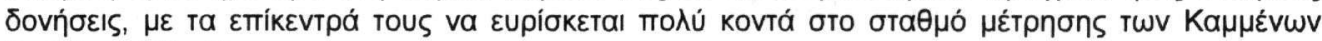
Boúp^wv (Пív. 2).

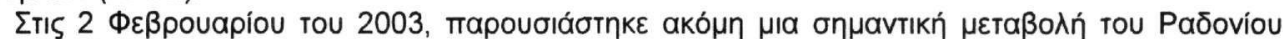

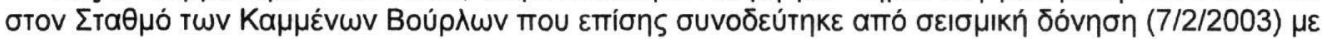
то їठı हті́кєVтро (Піv. 2).

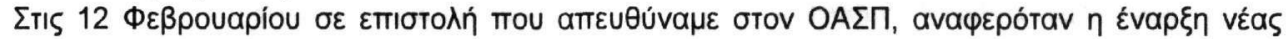

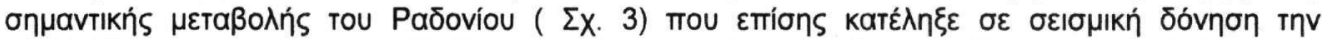

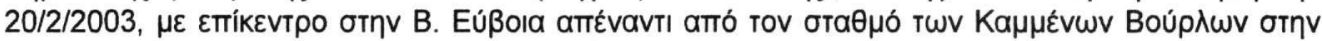

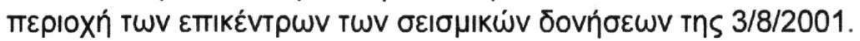

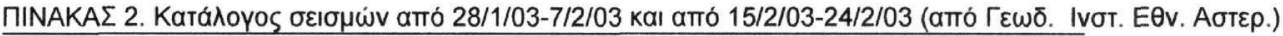

\begin{tabular}{lccccc}
\hline Date & $\begin{array}{c}\text { Time } \\
(\mathrm{GMT})\end{array}$ & $\begin{array}{c}\text { North } \\
(\mathrm{N})\end{array}$ & $\begin{array}{c}\text { East } \\
(\mathrm{E})\end{array}$ & $\begin{array}{c}\text { Depth } \\
(\mathrm{km})\end{array}$ & $\begin{array}{c}M \\
(\text { Local })\end{array}$ \\
\hline 2003 JAN 29 & 02136.3 & 38.70 & 22.75 & 5 & 2.8 \\
2003 JAN 29 & 02446.8 & 38.73 & 22.68 & 4 & 2.7 \\
2003 JAN 29 & 09093.9 & 38.75 & 22.82 & 13 & 3.1 \\
2003 FEB 7 & 130652.8 & 38.72 & 22.79 & 10 & 3.2 \\
2003 FEB 22 & 022316.3 & 38.74 & 23.47 & 23 & 3.5 \\
\hline
\end{tabular}




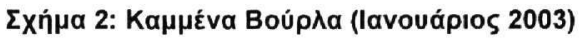

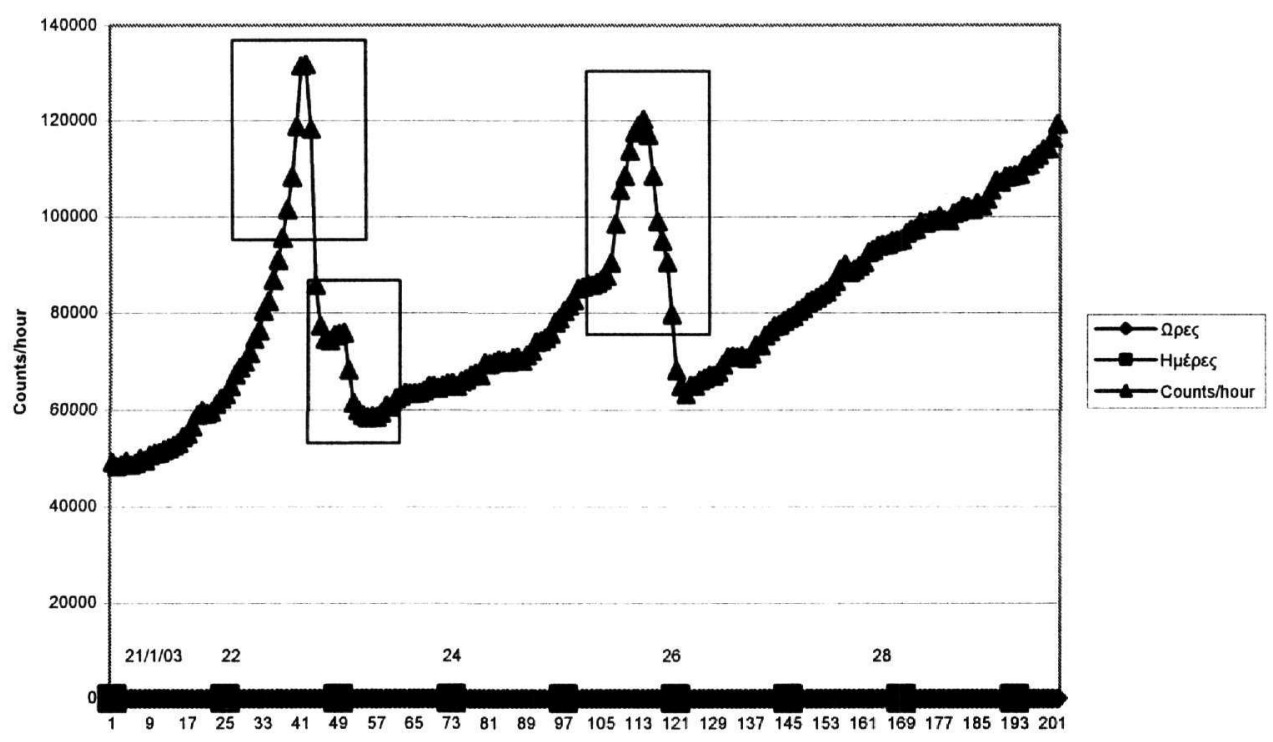

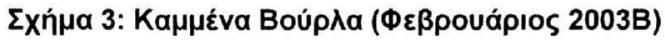

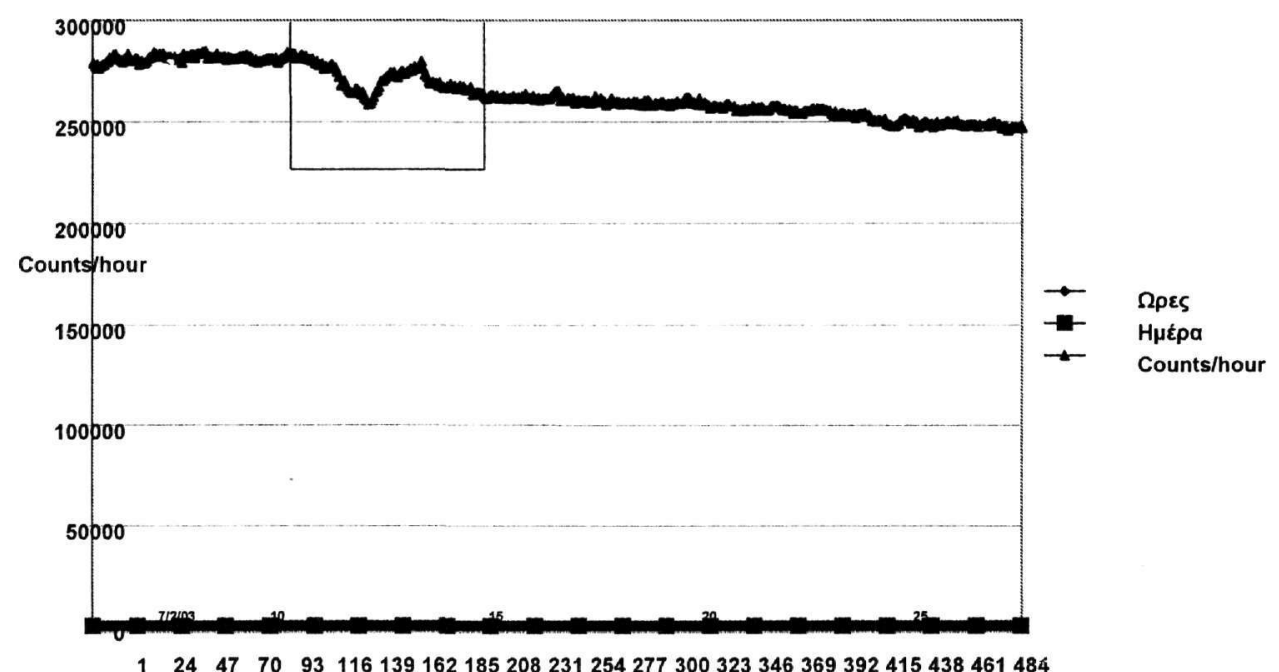

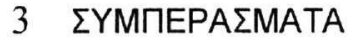

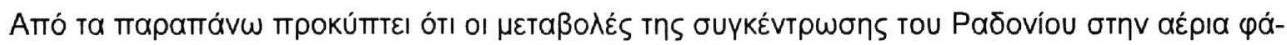

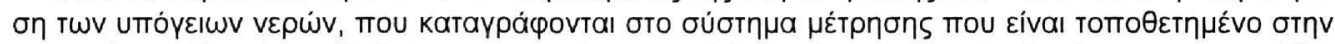

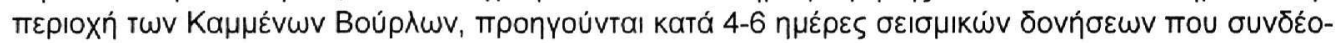

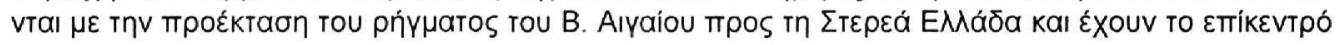




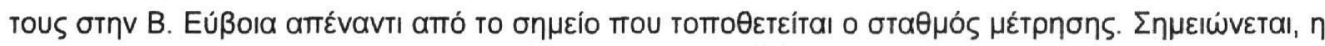

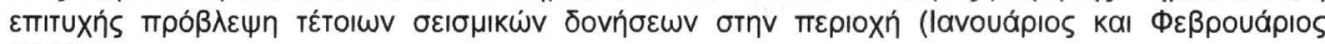
2003).

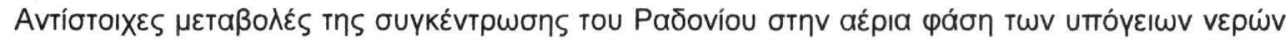

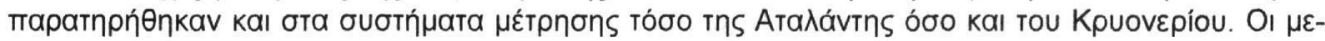

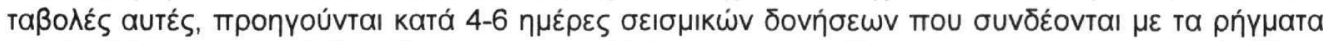

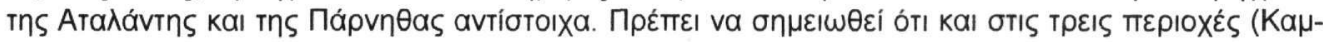

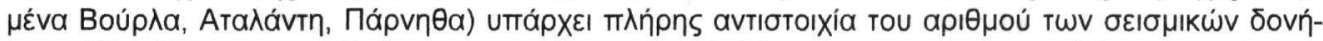

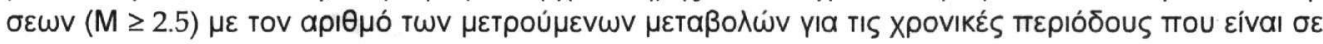

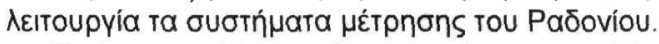

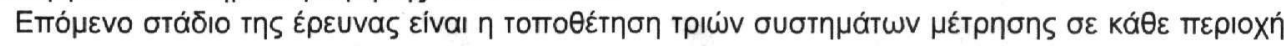

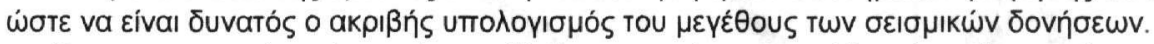

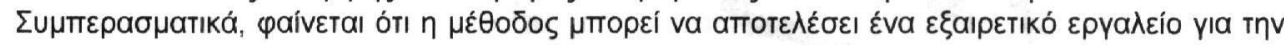

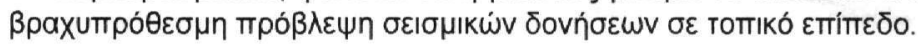

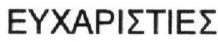

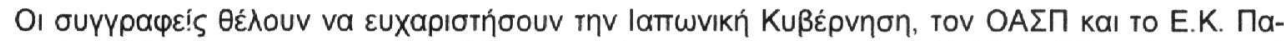

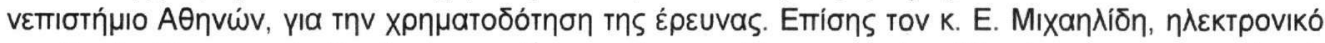

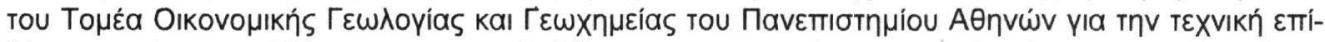

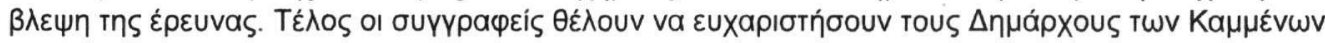

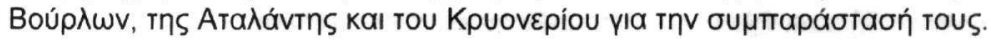

\section{ANAФOPE $\Sigma$}

Group of Hydrochemistry, Seismological Brigade of Hebei Province (1976). Studies of forecasting earthquakes in the light of the abnormal variations of Rn concentration in ground water. Acta Geophys. Sinica, 18, 279283.

Igarashi G. \& Wakita H. (1991). Tidal responses and earthquake-related changes in the water level of deep wells. J. Geophys. Res.,96, 4269-4278.

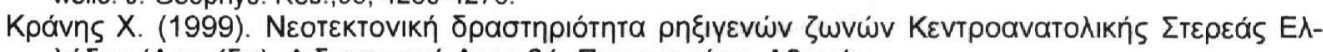

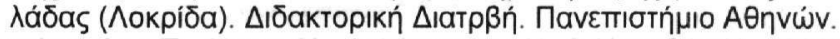

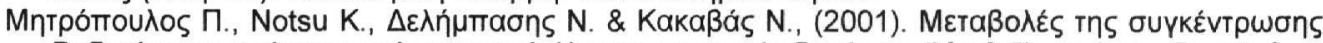

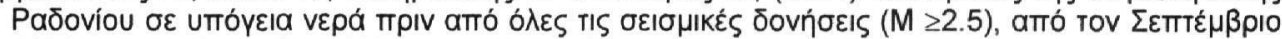

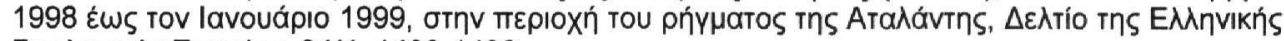

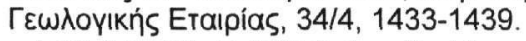

Noguchi M. \& Wakita H. (1977). A method for continuous Measurement of Radon in groundwater for earthquake prediction. J. Geophys. Res., 82, 1353-1357.

Notsu K., Wakita H., Igarashi G. \& Sato T. (1991). Hydrological and geochemical changes related to the 1989 seismic and volcanic activities off the Izu peninsula. J. Phys. Earth, 39, 245-254.

Ulomof V.I. \& Mavashev B.Z. (1971). The Tashkent earthquake of 26 April 1966. Akad. Nauk. Uzbek. SSR FAN, 188.

Wakita H., Nakamura Y., Notsu K., Noguchi M. \& Asada T. (1980). Radon anomaly: A possible precursor of the 1978 Izu-Oshima-kinkai Earthquake. Science, 207, 882-883.

Wakita H., Igarashi G., Nakamura Y., Sano Y. \& Notsu K., (1989). Coseismic Radon changes in groundwater. Geophys. Res. Lett., 16, 417-420.

Wakita H., Igarashi G. \& Notsu K. (1991)An anomalous Radon decrease in groundwater prior to an M6.0 earthquake: A possible precursor? Geophys. Res. Lett., 18, 629-632. 


\title{
EARTHQUAKE PREDICTION IN THE AREA OF N. EVIA ON THE BASIS OF GROUNDWATER RADON CONCENTRATION CHANGES
}

\author{
P. Mitropoulos ${ }^{1}$, K. Notsu ${ }^{2}$, N. Delibasis ${ }^{1}$, N. Kakavas ${ }^{3}$ and P. Voudouris ${ }^{1}$ \\ 1. University of Athens, Department of Geology \\ 2. University of Tokyo, Department of Earthquake Chemistry \\ ${ }^{3}$ I. G.M.E., Mesogion Str. 70, 11527 , Athens
}

Significant changes in the groundwater radon concentration, were observed on the 29/7/2003, at Kammena Vourla. The radon concentration changes can be attributed to the temporary enrichment of the groundwater by radon from the surrounding rocks due to the action of stress release or stress accumulation prior an earthquake. EPPO was immediately informed for the existence of these changes.

On the $3 / 8 / 2003$, a significant number of earthquakes in Northern Evia were recorded in an interval of a few hours. The epicentres of the earthquakes were located in the conceivable line between the epicentre of the strong earthquake of Skyros island (July 2001) and the site of the station of radon measurement at Kammena Vourla, on the extension of the Northern Aegean fault.

In the 23/1/2003, in the same station, 3 significant changes of radon concentration were measured EPPO was immediately informed for impending seismic activity in Northern Evia. On the $29^{\text {th }}$ of January three earthquakes were recorded. Their epicentres were located in N. Evia, close to the radon measurement. station.

On the 2/2/2003, a new significant radon change occurred at Kammena Vourla followed by an earthquake (7/2/2003). In the $12 / 2 / 2003$ in a letter that we addressed to the EPPO administration, the beginning of a new radon change was reported which also led to an earthquake with the same epicentre.

The changes that are recorded in the system of radon measurement of Kammena Vourla are precursors of earthquakes, related to the extension of the $\mathrm{N}$. Aegean fault towards the Sterea Hellas and have their epicentre in N. Evia. It seems that the method can be an important tool for local, short term earthquake prediction. 